\title{
Effect of thermocouple time constant on sensing of temperature fluctuations in a fast reactor subassembly
}

\author{
P. Sharma ${ }^{1}$, N. Murali $^{1}$, and T. Jayakumar ${ }^{2}$ \\ ${ }^{1}$ Instrumentation \& Control Group, Indira Gandhi Centre for Atomic Research, Kalpakkam-603102, India \\ ${ }^{2}$ Metallurgy \& Materials Group, Indira Gandhi Centre for Atomic Research, Kalpakkam-603102, India \\ Correspondence to: P. Sharma (paawan.sharma@gmail.com)
}

Received: 18 July 2013 - Revised: 21 November 2013 - Accepted: 24 January 2014 - Published: 20 February 2014

\begin{abstract}
Knowledge of temperature fluctuations in fast reactor subassembly is very important from a safety point of view. The time constant of thermocouples which are used for measuring coolant temperature in a fast reactor varies owing to various factors. Hence, it becomes necessary to investigate the effect of change in the time constant on sensed fluctuations. This paper investigates the dependence of temperature fluctuations on thermocouple time constants. A Scilab model consisting of source temperature profile, second-order thermocouple and histogram calculation is designed. Simulation is performed for various levels of fluctuations, fixed and variable thermocouple time constants. Kurtosis for each condition is calculated with the help of a histogram. It is found that the effect of true source fluctuations on sensor output is very large compared to that of a similar percentage of time-constant variations. Hence in systems like fast reactors, where the degree of source fluctuations (fluid enthalpy) is large in comparison to that of time-constant variations, the overall effect can be considered with great confidence to be the outcome of coolant temperature rather than thermocouple time-constant variations.
\end{abstract}

\section{Introduction}

A subassembly Srinivasan et al. (2006) consists of closely packed thin cylindrical tubes of fuel pellets. The coolant flows through a subassembly and transfers the heat generated due to fission. Thermocouple is placed at the subassembly outlet. The temperature reading so obtained fluctuates in nature. The analysis of such fluctuations is very important from a safety point of view. These fluctuations originate due to turbulent mixing of different temperature sodium streams across several fuel pins inside each fuel subassembly Vaidyanathan et al. (2006). The streams are at different temperatures because the fast neutron spectrum varies throughout the core, with a maximum value at the centre of the core and then gradually decreasing radially outwards. Even with arrangements for proper mixing of sodium, there exists a minimal amount of fluctuation for a particular geometry Gajapathy et al. (2007). The fluctuation level increases with overall reactor power as temperature also increases. The thermocouple time-constant value plays an important role in detecting these fluctuations, as smaller time constants are more efficient in recording them Donaldson et al. (2008). In order to study the factors affecting the fluctuations, a proper analysis is performed considering thermocouple parameters, source temperature and fluctuating time constants. The rest of the paper is organized as follows. Section 2 briefly describes the principle of thermocouple, the core arrangement, and subassembly structure. Recorded data from Fast Breeder Test Reactor (FBTR) at different power levels are discussed in Sect. 3. The simulation setup and methodology are explained in Sect. 4 followed by analysis and discussion in Sect. 5 .

\section{Sensor and setup}

The details about the sensor and its typical arrangement in a fast reactor subassembly are discussed here.

\subsection{Thermocouple}

Thermocouples are widely used in numerous industrial applications including nuclear reactors due to their range, ruggedness and accuracy. A second-order model of thermocouple 


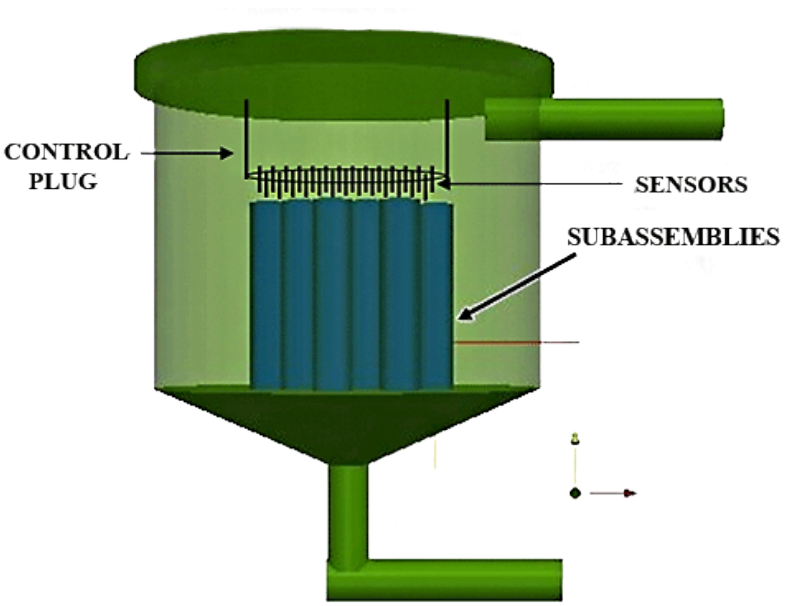

Figure 1. Basic structure of a fast reactor.

can be given as

$H(s)=\frac{1}{\tau s^{2}+s+1}$,

where $\tau$ is the thermocouple time constant (time taken to reach $63.2 \%$ of the final value). Thermocouple response time varies with various configurations such as directly exposed (few $\mathrm{ms}$ ), grounded sheathed $(\simeq 100 \mathrm{~ms})$, ungrounded sheathed (few $100 \mathrm{~ms}$ ), and inside thermowell (5-7 s). The Time constant depends on the mass of the thermocouple $(m)$, specific heat of thermocouple wire material $(c)$, convective heat transfer coefficient $(h)$ of the local medium and the surface area $(A)$ Bentley (1998) and is given as

$\tau=\frac{m c}{h A}$.

The bandwidth of a thermocouple is given as Hung et al. (2004)

$\omega_{B}=K d^{m-2} v^{m}$

where $K$ is an invariant constant, $m$ is a constant $(0.3 \leq m \leq 0.7), d$ is wire diameter and $v$ is coolant velocity. In terms of wire diameter, $\tau$ is represented as Tagawa and Ohta (1997)

$\tau=\frac{\rho c d}{4 h}=\frac{\rho c d^{2}}{4 N u \lambda_{g}}$,

where $\rho$ is wire material density, $N u$ is Nusselt's number, and $\lambda_{g}$ is thermal conductivity of fluid around thermocouple. $N u$ is a function of Reynolds number, Grashoff number and Prandtl number.

$N u=f(\operatorname{Re}, G r, P r)$

It is clear from Eqs. (2), (4) and (5) that $\tau$ is dependent on many parameters involving the construction and geometry of the sensor as well as the operating conditions. Equation (3) shows that effective bandwidth offered by a thermocouple depends on coolant flow, which in turn affects $\tau$.

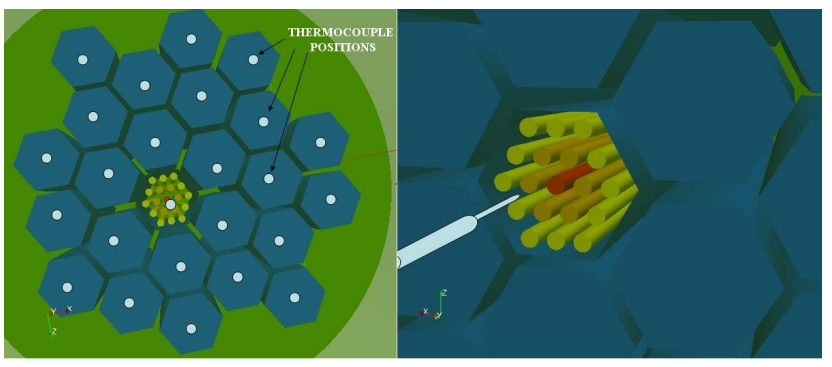

Figure 2. Subassembly arrangement.

A proper understanding of the whole arrangement is required for analysing the time constant in the case of a fast reactor subassembly.

\subsection{Fast reactor subassembly}

A very basic arrangement of a typical loop type fast reactor is shown in Fig. 1. The main feature of a loop type reactor is that the coolant pump is located outside the main vessel.

A group of cylindrical fuel pins is placed inside a hexagonal tube called subassembly as shown in Fig. 2.

The temperature profile inside each subassembly as well as amongst all subassemblies is not uniform since the neutron spectrum of the core peaks at the core centre and decreases radially outwards Waltar and Reynolds (1981). The fuel pins in a subassembly are at different temperatures. Coolant streams of different temperature coming out of a subassembly get mixed and form a fluctuating profile. For proper mixing of coolant streams, each fuel pin is covered with a spacer wire which also acts as a separator between different fuel pins. However, with all the provisions for better mixing, there exists a minimum amount of fluctuation level in temperature profiles. The fluctuation level depends on the overall reactor power. The centre-most subassembly is known as the central subassembly(CSA). The CSA contributes maximally to the reactor power. Thermocouples are located above each subassembly outlet and submerged in coolant. For CSA, sheathed thermocouples are used for faster response (around $100 \mathrm{~ms}$ ), whereas they are used with thermowell in all other subassemblies (5-6s) for a longer life time of the sensor. Figure 2 shows the top view of hexagonal subassemblies arranged compactly. Each subassembly consists of a fixed number of cylindrical fuel pins. The actual structure is however complex with more features, and only related details are shown here for a simple understanding.

\section{Reactor data}

Experimental data from the FBTR are used as an aid for developing a signal analysis methodology. The various states of a reactor are shut down, reactor startup, reactor under operation (ROP), fuel handling startup and fuel handling. The 


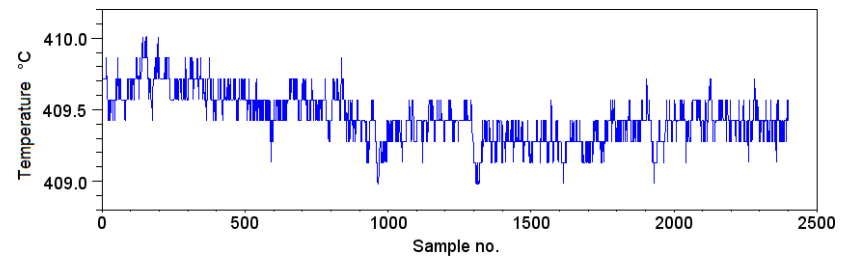

(a)

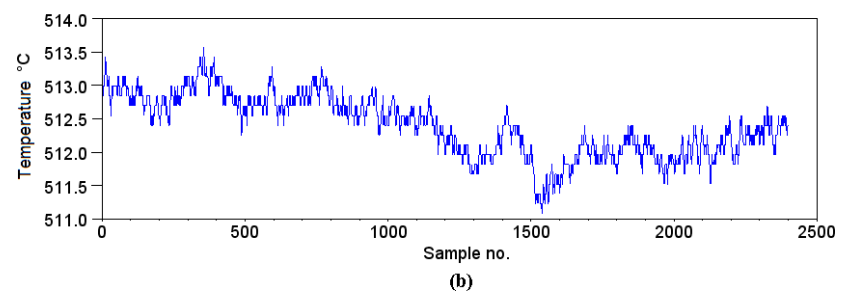

Figure 3. CSA temperature profile at stable power levels (a) $11 \mathrm{MWTh}$ and (b) $18 \mathrm{MWTh}$.

CSA temperature profile, along with other important parameters, was collected for all discrete power levels in $R O P$ state. Figure 3 shows the CSA temperature profile for two stable power levels of $11 \mathrm{MWTh}$ and $18 \mathrm{MWTh}$ (thermal).

Figure 4 shows the CSA temperature profile for gradually increasing power level.

It is clear from these figures that the amount of fluctuation increases with thermal power for nearly constant coolant flow $\left(328-334 \mathrm{~m}^{3} \mathrm{~h}^{-1}\right)$.

The parameters which are used for modelling and simulation are mean temperature value $(\mu)$ and corresponding standard deviation $(\sigma), \sigma$ represents the degree of fluctuation. Hence a higher power level would have a high values of $\mu$ and $\sigma$. It is to be noted that, though $\sigma$ increases with power, it is non-linearly localized in time since the source of such fluctuations is due to mixing of highly turbulent coolant streams passing through a subassembly.

The value of $(\mu, \sigma)$ is $(409.44,0.17168)^{\circ} \mathrm{C}$ for $11 \mathrm{MWTh}$ and $(512.39,0.45812)^{\circ} \mathrm{C}$ for $18 \mathrm{MWTh}$. Table 1 represents unique $(\mu, \sigma)$ for increasing reactor power. The time series is made stationary by performing first-order differencing. This differenced series is added to the overall mean of actual series. If $a$ is the original time series of 2400 samples taken 10 samples per second, $b$ being the differenced series (2399 samples), stationary time series $c$ is calculated by Eqs. (6), (7) and (8).

$c=\mu+\sigma$

$\mu=(2400)^{-1} \sum_{i=1}^{2400} a_{i}$

$\sigma=b_{i}=\operatorname{diff}(a)$

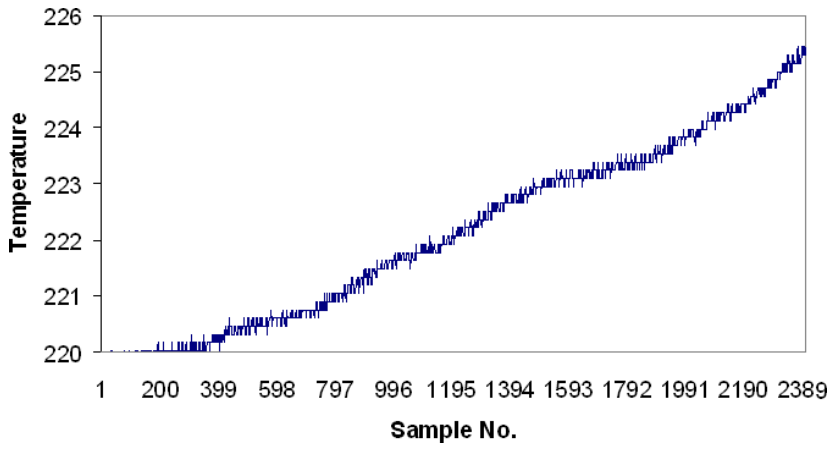

Figure 4. CSA temperature profile for increasing power.

Table 1. Reactor temperature and temperature fluctuations as a function of the reactor power.

\begin{tabular}{lr}
\hline$\mu$ & Recalculated $\sigma$ \\
\hline 221.86 & 0.091 \\
231.3 & 0.093 \\
249.08 & 0.095 \\
365.48 & 0.097 \\
378.66 & 0.102 \\
409.44 & 0.171 \\
512.39 & 0.458 \\
\hline
\end{tabular}

\section{Simulation methodology}

By using the collected reactor data, a simulation model is proposed as shown in Fig. 5.

The type $\mathrm{K}$ thermocouple is modelled for various values of $\tau$ ranging from 0.05 to $0.5 \mathrm{~s}$ using polynomial coefficients from NIST database (NIST, 2004). Based on the reactor data, the source temperature profile with fixed mean and standard deviation is obtained using the grand function of Scilab SCILAB (2013). The mean value is fixed at $300^{\circ} \mathrm{C}$ for a closer approach towards analysis of the effect of variable fluctuations in the source (coolant) $(\sigma)$ itself.

The following conditions were analysed for fixed $\mu$ to observe their relative effect:

- Various constant values of $\sigma$ and $\tau$

- Various fluctuating levels of $\sigma$ and $\tau$

Response to fluctuating (variable) values of $\tau$ is calculated by multiplexing even and odd terms of two different $\tau$ response values. The related Scilab scripts and function codes can be referenced from codes.

A histogram plot gives frequency versus variable information of the data and is performed for the calculation of mean and standard deviation from the simulated sensor output. From these two parameters, kurtosis is calculated, which gives an estimate of probability distribution (frequency distribution) of the data. So for all the test conditions, data 


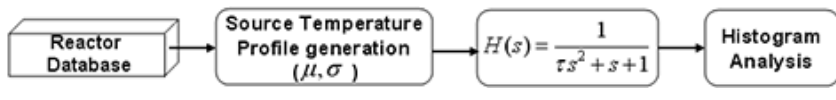

Figure 5. Simulation model.

profiles are simulated and kurtosis is calculated by using histogram.

The mean $\left(\mu_{h}\right)$, standard deviation $\left(\sigma_{h}\right)$ and kurtosis $\left(\beta_{2}\right)$ are calculated as follows.

$\mu_{h}=\frac{1}{n} \sum_{i=1}^{n} f_{i} x_{i}$

$\sigma_{h}=\sqrt{\frac{1}{n} \sum_{i=1}^{n} f_{i}\left(x_{i}-\mu\right)^{2}}$

$\beta_{2}=\frac{\sum_{i=1}^{n}\left(X_{i}-\mu\right)^{4}}{(n-1) \sigma^{4}}$

Maximum possible value of kurtosis is given as

$\beta_{2, \max }=\frac{(n-1)^{3}-1}{n(n-1)}$.

Many works have been reported on the use of kurtosis in signal processing, such as Picard (1951), Vrabie et al. (2004) and Nopiah et al. (2009). The main characteristic of kurtosis is that it accurately depicts the peak and distribution of the data.

Any change in the distribution of observation data would reflect in a different $\beta_{2}$ value. The idea is to observe the relative effect on $\beta_{2}$ for $\pm x \%$ fluctuations in $\sigma$, keeping $\tau$ constant and vice versa - i.e. to observe the effect on $\beta_{2}$ for $\pm x \%$ fluctuations in $\tau$, keeping $\sigma$ constant. In this way, a relative analysis is possible to quantify the individual effect brought on by $\sigma$ and $\tau$ separately on the sensed signal.

\section{Results and discussion}

Simulation results are obtained to study the effect of various combinations of parameters on the fluctuations. Various functions written in Scilab are mentioned below.

\section{- musigmakurtosis(arg1)}

Returns data length $(N), \mu_{h}, \sigma_{h}, \beta_{2}$ and $\beta_{2, \max }$ for a fixed mean value of source thermal profile $\left(300^{\circ} \mathrm{C}\right)$, and standard deviation (fluctuation level $\sigma$ ) given by $\arg 1$.

\section{- skewkurt(arg1,arg2)}

Returns data length $(N), \mu_{h}, \sigma_{h}, \beta_{2}$ and $\beta_{2, \max }$ for a source thermal profile given by arg 2 and $\tau$ given by argl.
Table 2. $\beta_{2}$ vs. $\sigma, \mu=300^{\circ} \mathrm{C}, \tau=0.1 \mathrm{~s}$.

\begin{tabular}{lr}
\hline$\sigma$ & $\beta_{2}$ \\
\hline 0.05 & 0.087 \\
0.1 & 0.350 \\
0.15 & 1.059 \\
0.2 & 1.819 \\
0.25 & 2.270 \\
0.3 & 3.893 \\
\hline
\end{tabular}

Table 3. $\beta_{2}$ vs. $\tau, \mu=300{ }^{\circ} \mathrm{C}, \sigma=0.1^{\circ} \mathrm{C}$.

\begin{tabular}{lr}
\hline$\tau$ & $\beta_{2}$ \\
\hline 0.05 & 0.416 \\
0.1 & 0.425 \\
0.15 & 0.434 \\
0.2 & 0.443 \\
0.25 & 0.451 \\
0.3 & 0.459 \\
\hline
\end{tabular}

- flsigma(arg1, arg2)

Returns a fluctuating source thermal profile with limits between $\arg 1$ and $\arg 2$, with fixed value of $\mu\left(300^{\circ} \mathrm{C}\right)$.

\section{- $f$ tau(arg1, arg2,arg3)}

Returns response to a given source $\arg 1$ with $\tau$ fluctuating between values given by $\arg 2$ and $\arg 3$.

Table 2 indicates the variation of $\beta_{2}$ with $\sigma$. Similarly, Table 3 gives the variation of $\beta_{2}$ with $\tau$. It is clear that variations in source (coolant) temperature have greater impact on $\beta_{2}$.

Figure 6 shows the kurtosis variation for different values for $\tau$ and $\sigma$.

For analysing variable $\sigma$ and $\tau$ (i.e. both source profile as well as thermocouple time constant tend to fluctuate due to reactor thermodynamic conditions), various levels of source and time-constant fluctuations are generated by specifying the minimum and maximum threshold values.

For example, a source profile with fluctuation levels between 0.1 and 0.2 can be generated in Scilab as

$a=f l \_\operatorname{sigma}(0.1,0.2)$.

Similarly, thermocouple response to a source profile (either constant or fluctuating) with time constant varying between 0.1 and $0.2 \mathrm{~s}$ can be obtained as

$b=f l$ tau(source profile, $0.1,0.2$ ).

Results for various combinations of source and time-constant behaviour are shown in Figs. 7 and 8.

The mean value of $\sigma$ and $\tau$ are fixed at $0.1{ }^{\circ} \mathrm{C}$ and $0.1 \mathrm{~s}$ respectively. Fluctuation levels of 5-35\% around these mean values were simulated in different cases. 


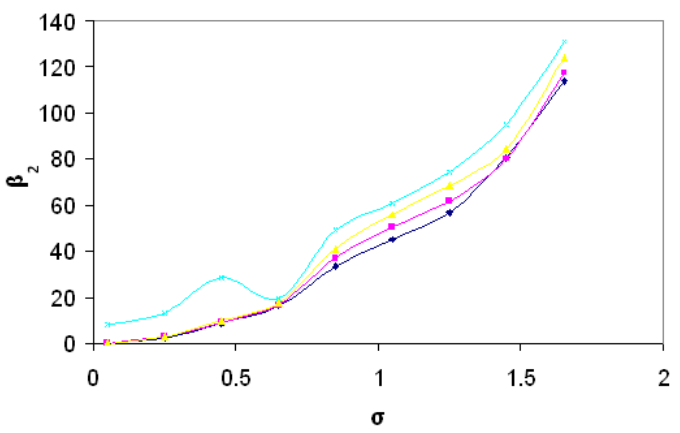

Figure 6. $\beta_{2}$ vs. $\sigma$.

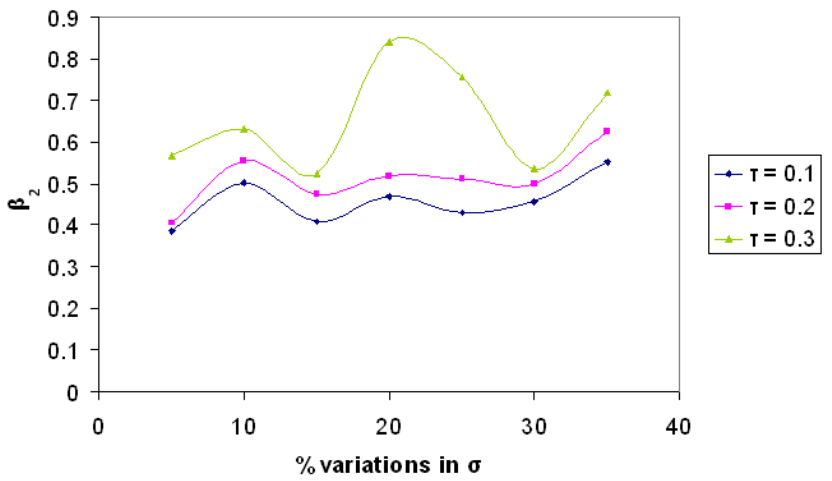

Figure 7. $\beta_{2}$ vs. $\%$ variations in $\sigma$.

To simulate a more realistic condition, where both source and time constant tend to vary, both $\sigma$ and $\tau$ are allowed to swing between fixed levels in many possible combinations for, e.g., $5 \%$ fluctuation in $\sigma$ with $\pm(5,15,25,35) \%$ fluctuations in $\tau$ and vice versa. The result is shown in Fig. 9.

The cases analysed till now are comprises of values varying around mean values of $\sigma=0.1{ }^{\circ} \mathrm{C}$ and $\tau=0.1 \mathrm{~s}$. Result for the values of $\sigma, \tau$ constant and analysed with regard to fluctuating levels mutually are shown in Fig. 10. It is clear from Fig. 10 that the degree of change in kurtosis is far greater for fluctuating $\sigma$ than that of fluctuating $\tau$; i.e. fluctuations observed in thermocouple readings are largely due to the fluctuations in the source (coolant) itself and hence a good indicator of coolant condition with time.

Any change in kurtosis value denotes a shift in the signal properties by means of the frequency distribution. In a fast reactor, where the mean temperature level as well as the associated fluctuations increases with thermal power, kurtosis acts as an indicator of change in the frequency distribution of the signal. The observation that kurtosis increases with $\sigma$ explains why the fluctuations at higher power level attain a sharper and narrow distribution. It means that the core temperature signal is broadly distributed with lesser peakedness at low thermal power than at higher power. These results give sufficient confidence in estimating parameters based on

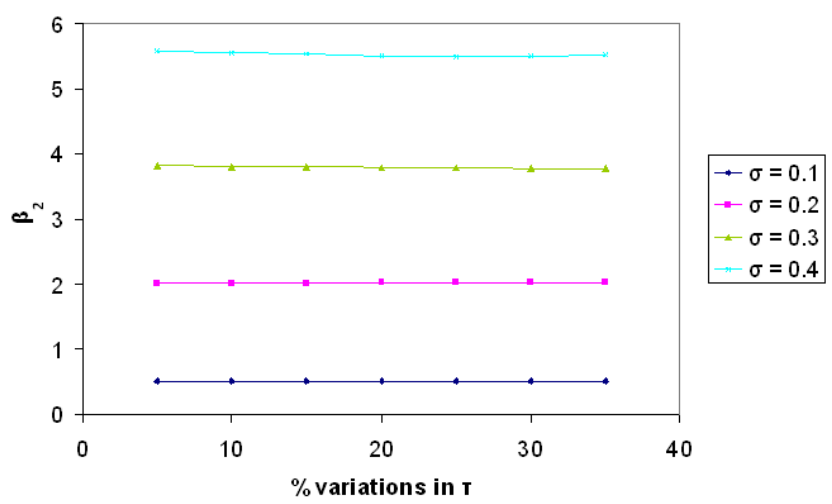

Figure 8. $\beta_{2}$ vs. $\%$ variations in $\tau$.

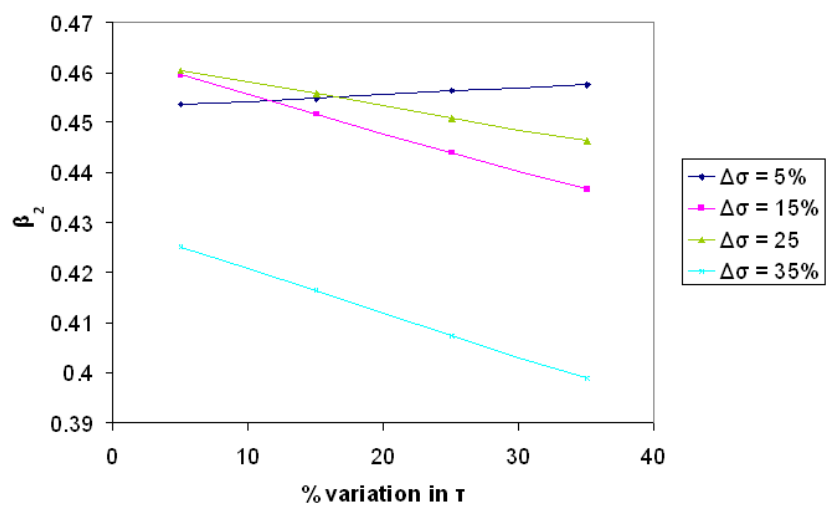

Figure 9. $\beta_{2}$ vs. $\%$ variations in $\tau$ and $\sigma$.

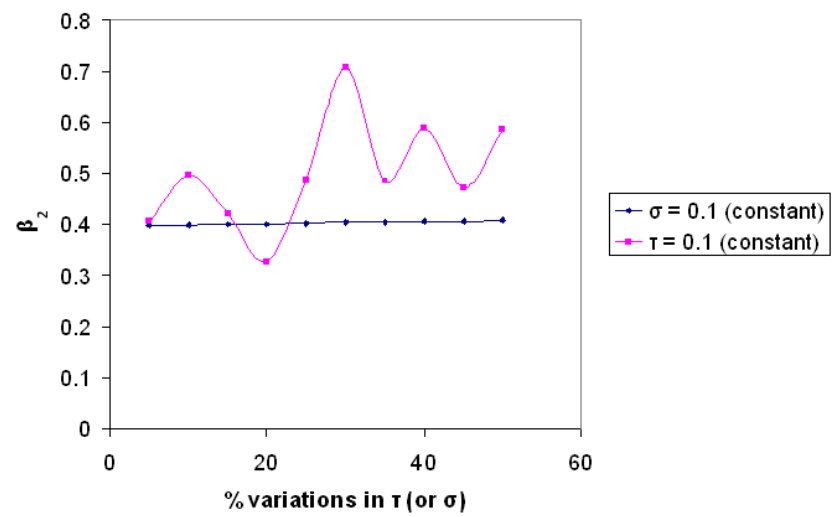

Figure 10. $\beta_{2}$ vs. $\%$ variations in $\tau$ or $\sigma$.

temperature fluctuations. Also, it supports the idea of using fluctuations in spite of its non-stationarity. There are ways by which the non-stationarity can be reduced, like first-order differencing. 


\section{Conclusions}

Variations in thermocouple time constant over time were simulated and studied in arrangement with different power levels and fluctuations. It is found that the effect of time-constant variations on sensor readings is much less in comparison to similar variations in source (coolant) temperature. Hence, parameters calculated on the basis of source fluctuations gives very accurate information about source thermodynamic condition, even in the presence of the sensor's time-constant variations. The simulation codes can be used for analysis from the link. [https://sites.google.com/ site/paawankuvera/home/kurtosisanalysis]

Edited by: G. S. Aluri

Reviewed by: two anonymous referees

\section{References}

Bentley, R. E. (Ed.): Handbook of Temperature Measurement Vol. 3: The Theory and Practice of Thermoelectric Thermometry, Vol. 3, Springer, 1998.

Donaldson, A. B., Lucero, R. E., Gill, W., and Yilmaz, N.: Problems encountered in fluctuating flame temperature measurements by thermocouple (No. SAND2008-7384J), Sandia National Laboratories, 2008.

Gajapathy, R., Velusamy, K., Selvaraj, P., Chellapandi, P., and Chetal, S. C.: CFD investigation of helical wire-wrapped 7-pin fuel bundle and the challenges in modeling full scale 217 pin bundle, Nucl. Eng. Des., 237, 2332-2342, 2007.

Hung, P., McLoone, S., Irwin, G. W., and Kee, R. J.: A total least squares approach to sensor characterisation, System Identification 2003, 321-324, 2004.
NIST: Thermocouple - NIST ITS-90 Thermocouple Database, available at: http://srdata.nist.gov/its90/download/type_k.tab (last access: 16 July 2013), 2004.

Nopiah, Z. M., Khairir, M. I., Abdullah, S., Nizwan, C. K. E., and Baharin, M. N.: Peak-valley segmentation algorithm for Kurtosis analysis and classification of fatigue time series data, Eur. J. Sci. Res., 29, 113-125, 2009.

Picard, H. C.: A note on the maximum value of kurtosis, Ann. Math. Stat., 22, 480-482, 1951.

SCILAB: Random number generator(s), SCILAB, available at: http://help.scilab.org/docs/5.3.0/en_US/grand.html (last access: 16 July 2013), 2013.

Srinivasan, G., Suresh Kumar, K. V., Rajendran, B., and Ramalingam, P. V.: The fast breeder test reactor-design and operating experiences, Nucl. Eng. Des., 236, 796-811, 2006.

Tagawa, M. and Ohta, Y.: Two-thermocouple probe for fluctuating temperature measurement in combustion - Rational estimation of mean and fluctuating time constants, Combust. Flame, 109, 549-560, 1997.

Vaidyanathan, G., Kasinathan, N., and Velusamy, K.: Dynamic model of Fast Breeder Test Reactor, Ann. Nucl. Energy, 37, 450462, 2010.

Vrabie, V., Granjon, P., Maroni, C. S., and Leprettre, B.: Application of spectral kurtosis to bearing fault detection in induction motors, in: Proceedings of the 5th International Conference on acoustical and vibratory surveillance methods and diagnostic techniques, 11-13 October 2004, Senlis, France, 2004.

Waltar, A. E. and Reynolds, A. B.: Fast breeder reactors, Pergamon, 1981. 